\title{
Thrombocytopenia is associated with severe retinopathy of prematurity
}

\author{
Bertan Cakir,, ${ }^{1,2}$ Raffael Liegl,,${ }^{1,3}$ Gunnel Hellgren, ${ }^{4}$ Pia Lundgren, ${ }^{4}$ Ye Sun, ${ }^{1}$ Susanna Klevebro, ${ }^{5}$ \\ Chatarina Löfquist, ${ }^{4}$ Clara Mannheimer, ${ }^{4}$ Steve Cho, ${ }^{1}$ Alexander Poblete, ${ }^{1}$ Rubi Duran, ${ }^{1}$ \\ Boubou Hallberg, ${ }^{5}$ Jorge Canas, ${ }^{6}$ Viola Lorenz, ${ }^{6}$ Zhi-Jian Liu, ${ }^{6}$ Martha C. Sola-Visner, ${ }^{6}$ \\ Lois E.H. Smith, ${ }^{1}$ and Ann Hellström ${ }^{4}$ \\ 'Department of Ophthalmology, Boston Children's Hospital, Harvard Medical School, Boston, Massachusetts, USA. \\ ${ }^{2}$ Eye Center, Medical Center, Faculty of Medicine, University of Freiburg, Germany. ${ }^{3}$ Department of Ophthalmology, Ludwig- \\ Maximilians-University, Munich, Germany. ${ }^{4}$ Section for Ophthalmology, Department of Clinical Neuroscience, Institute \\ of Neuroscience and Physiology, Sahlgrenska Academy, University of Gothenburg, Gothenburg, Sweden. ${ }^{5}$ Department of \\ Neonatology, Karolinska Institutet and University Hospital, Stockholm, Sweden. ${ }^{6}$ Department of Pediatrics, Division of \\ Newborn Medicine, Boston Children's Hospital, Harvard Medical School, Boston, Massachusetts, USA.
}

Retinopathy of prematurity (ROP) is characterized by abnormal retinal neovascularization in response to vessel loss. Platelets regulate angiogenesis and may influence ROP progression. In preterm infants, we assessed ROP and correlated with longitudinal postnatal platelet counts $(n=202)$. Any episode of thrombocytopenia $\left(<100 \times 10^{9} / I\right)$ at $\geq 30$ weeks postmenstrual age (at onset of ROP) was independently associated with severe ROP, requiring treatment. Infants with severe ROP also had a lower weekly median platelet count compared with infants with less severe ROP. In a mouse oxygen-induced retinopathy model of ROP, platelet counts were lower at P17 (peak neovascularization) versus controls. Platelet transfusions at P15 and P16 suppressed neovascularization, and platelet depletion increased neovascularization. Platelet transfusion decreased retinal of vascular endothelial growth factor A (VEGFA) mRNA and protein expression; platelet depletion increased retinal VECFA mRNA and protein expression. Resting platelets with intact granules reduced neovascularization, while thrombin-activated degranulated platelets did not. These data suggest that platelet releasate has a local antiangiogenic effect on endothelial cells to exert a downstream suppression of VEGFA in neural retina. Low platelet counts during the neovascularization phase in ROP is significantly associated with the development of severe ROP in preterm infants. In a murine model of retinopathy, platelet transfusion during the period of neovascularization suppressed retinopathy.

Authorship note: BC, RL, CH, and PL contributed equally to this work.

Conflict of interest: The authors have declared that no conflict of interest exists.

Submitted: December 26, 2017

Accepted: August 23, 2018

Published: October 4, 2018

Reference information: JCI Insight. 2018;3(19):e99448. https://doi.org/10.1172/jici. insight.99448.

\section{Introduction}

Retinopathy of prematurity (ROP) is a potentially blinding disease of preterm infants (1), characterized by abnormal vascularization in the maturing retina. After preterm birth, low levels of insulin-like growth factor (IGF-I) and hyperoxia-induced suppression of vascular endothelial growth factor A (VEGFA) (2) suppress normal retinal vascularization. The avascular hypoxic retina induces VEGFA, stimulating vascular proliferation first detectable at 30-32 weeks postmenstrual age (PMA) (3).

Platelets are important in angiogenesis regulation and may influence the pathogenesis of ROP $(4,5)$. Thrombocytopenia is common in preterm infants $(6,7)$ and is frequently associated with sepsis and necrotizing enterocolitis (NEC) $(8,9)$. A case report suggests that platelet transfusion during proliferative ROP might inhibit neovascularization (NV) (10). Studies have shown an association between severe ROP and thrombocytopenia in neonatal life (10-14), but the influence of platelets in animal models of ROP has not been reported. In preterm infants born at gestational age $(\mathrm{GA})<27$ weeks $(n=202)$, we correlated ROP with platelet counts from birth to $36 \mathrm{PMA}$ and found a correlation between thrombocytopenia in phase II (neovascular ROP) and the severity of ROP. To determine if platelet transfusion during this phase of ROP might suppress ROP development, we evaluated platelet counts in mice with oxygen-induced retinopathy (OIR) compared with controls and tested the effects of platelet transfusions (with and without granules) and platelet depletion on retinopathy. 
Table 1. Correlation of thrombocytopenia with severe ROP (requiring laser treatment) and no or less severe ROP (no treatment required)

\begin{tabular}{|c|c|c|c|c|c|c|c|}
\hline \multirow[b]{2}{*}{ Platelet variables } & \multirow[b]{2}{*}{$\begin{array}{c}\text { No ROP treatment } \\
\qquad(n=153)\end{array}$} & \multirow[b]{2}{*}{$\begin{array}{l}\text { ROP treatment } \\
(n=49)\end{array}$} & \multirow[b]{2}{*}{ p-value } & \multicolumn{4}{|c|}{ Logistic regression analyses } \\
\hline & & & & $\begin{array}{c}\text { OR } \\
(95 \% \mathrm{Cl})\end{array}$ & p-value & $\begin{array}{l}\text { OR adjusted }{ }^{A} \\
(95 \% \mathrm{Cl})\end{array}$ & p-value \\
\hline $\begin{array}{l}\text { Any early thrombocytopenia } \\
\text { (<30 weeks PMA) } \\
<100 \times 10^{9} / \mathrm{L}, \% \text { (n) }\end{array}$ & $56 \%(85 / 153)$ & $76 \%$ (37/49) & 0.013 & $\begin{array}{c}2.47 \\
(1.20-5.09)\end{array}$ & 0.015 & $\begin{array}{c}1.62 \\
(0.73-3.63)\end{array}$ & 0.24 \\
\hline $\begin{array}{l}\text { Any later thrombocytopenia } \\
(\geq 30 \text { weeks PMA) } \\
<100 \times 10^{9} / \mathrm{L}, \% \text { (n) }\end{array}$ & $16 \%(25 / 153)$ & $45 \%(22 / 49)$ & $<0.001$ & $\begin{array}{c}4.17 \\
(2.06-8.46)\end{array}$ & $<0.001$ & $\begin{array}{c}2.97 \\
(1.37-6.46)\end{array}$ & 0.006 \\
\hline
\end{tabular}

Abbreviations: GA, gestational age; NEC, necrotizing enterocolitis; PMA, postmenstrual age; ROP, retinopathy of prematurity

For categorical variables $\mathrm{n}(\%)$ is presented.

For comparison between groups Chi-square test was used.

Aadjusted for GA (week), NEC and sepsis

We defined the initial phase of ROP, with suppressed vascularization, as < 30 weeks PMA and the later proliferative phase of ROP from 30 to 36 weeks PMA. Sepsis was defined as positive blood culture and/or clinical signs and C-reactive protein $>20 \mathrm{mg} / \mathrm{L}$.

\begin{abstract}
Results
Clinical study. In our retrospective clinical study of 202 very low-birth weight infants, $24 \%(49 / 202)$ had severe ROP requiring laser photocoagulation (treatment). Mean GA at birth was 25.3 weeks (SD \pm 1.0 ), and mean birth weight was 782 grams (SD \pm 167). In univariable logistic regression analysis, the most prominent factor associated with severe ROP was any episode of thrombocytopenia at $\geq 30$ weeks PMA (odds ratio [OR] $=4.17$, 95\% CI, 2.06-8.46, $P<0.001$, Table 1). After adjusting for well-known confounding factors (GA, NEC, and sepsis), any episode of thrombocytopenia at the onset of proliferative retinopathy ( $\geq 30$ weeks PMA) persisted as a prominent independent association factor $(\mathrm{OR}=2.97,95 \% \mathrm{CI}, 1.37-6.46, P=0.006)$. Mean weekly platelet counts per infant were lower in infants treated for severe ROP compared with infants with no or less severe disease, at almost all postnatal weeks (Figure 1). The difference in mean weekly platelet counts further increased at $\geq 30$ weeks PMA in the proliferative ROP phase (Figure 1 ). All treated infants with any thrombocytopenia at $\geq 30$ weeks PMA also had some thrombocytopenia in the initial vessel loss phase of ROP ( $<30$ weeks PMA).
\end{abstract}

A

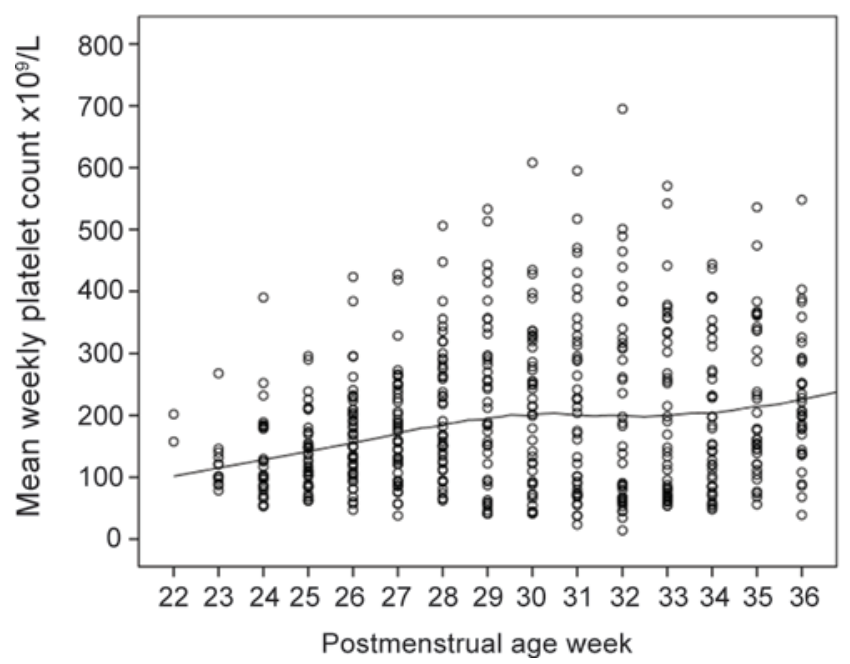

B

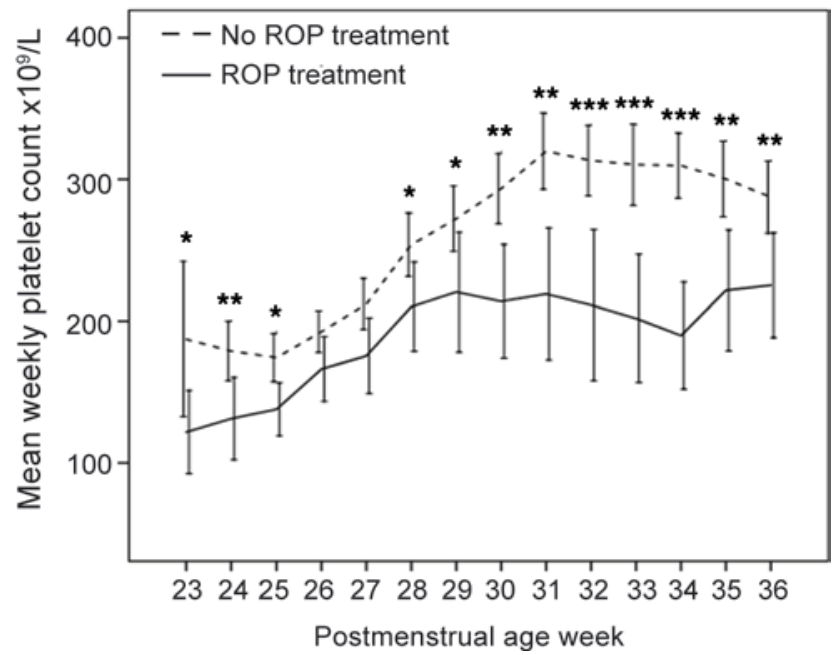

Figure 1. Platelet count and severity of retinopathy of prematurity. (A) Platelet counts of all infants screened for retinopathy of prematurity (ROP) per postmenstrual age week (PMA) (each dot represents the mean weekly platelet count of each individual; the black solid line represents the mean platelet count of all individuals) $(n=202)$. (B) Mean PMA platelet count $\left(1 \times 10^{9} / \mathrm{I}\right)$ of all infants with severe ROP requiring laser treatment ( $n=49$; solid line) and with no or less severe ROP not requiring laser treatment ( $n=153$; dashed line). Mean weekly platelet counts per infant were lower in infants treated for severe ROP compared with infants with no or less severe disease, at almost all postnatal weeks. The difference in mean weekly platelet counts further increased at $\geq 30$ weeks $P M A$ in the proliferative ROP phase. All treated infants with any thrombocytopenia at $\geq 30$ weeks PMA also had some thrombocytopenia in the initial vessel-loss phase of ROP (<30 weeks PMA). ${ }^{*} P<0.05,{ }^{* *} P<0.01$, ${ }^{* *} P<0.001$. Error bars represent $95 \% \mathrm{Cl}$. Mann-Whitney $U$ test was used for statistical analysis. 
A

\begin{tabular}{|c|c|c|} 
A & \multicolumn{1}{|c|}{} \\
\hline Ro & \multicolumn{1}{|c|}{} \\
\hline & P5\% Oxygen & P15 P16 P17 \\
\hline Vaso-obliteration & Rasoproliferation Air \\
\hline Phase I & Phase II
\end{tabular}

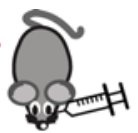

GPIb $\alpha$ antibody on P15

or isolated platelet on P15 and P16
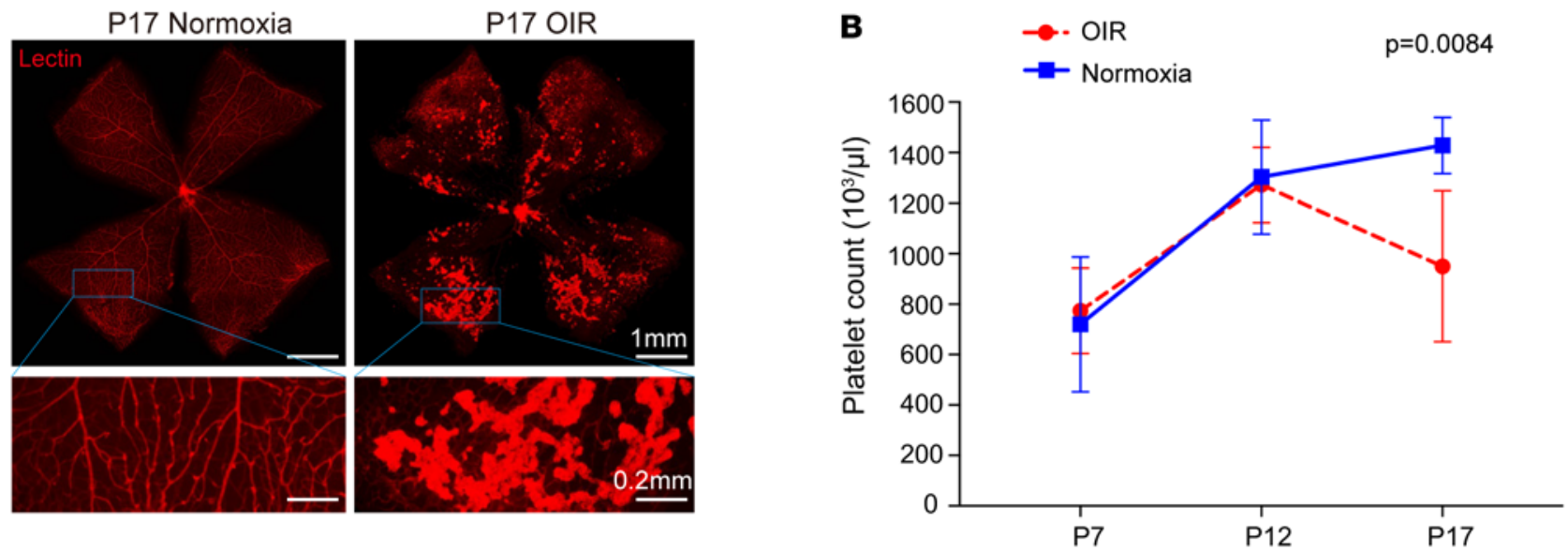

C
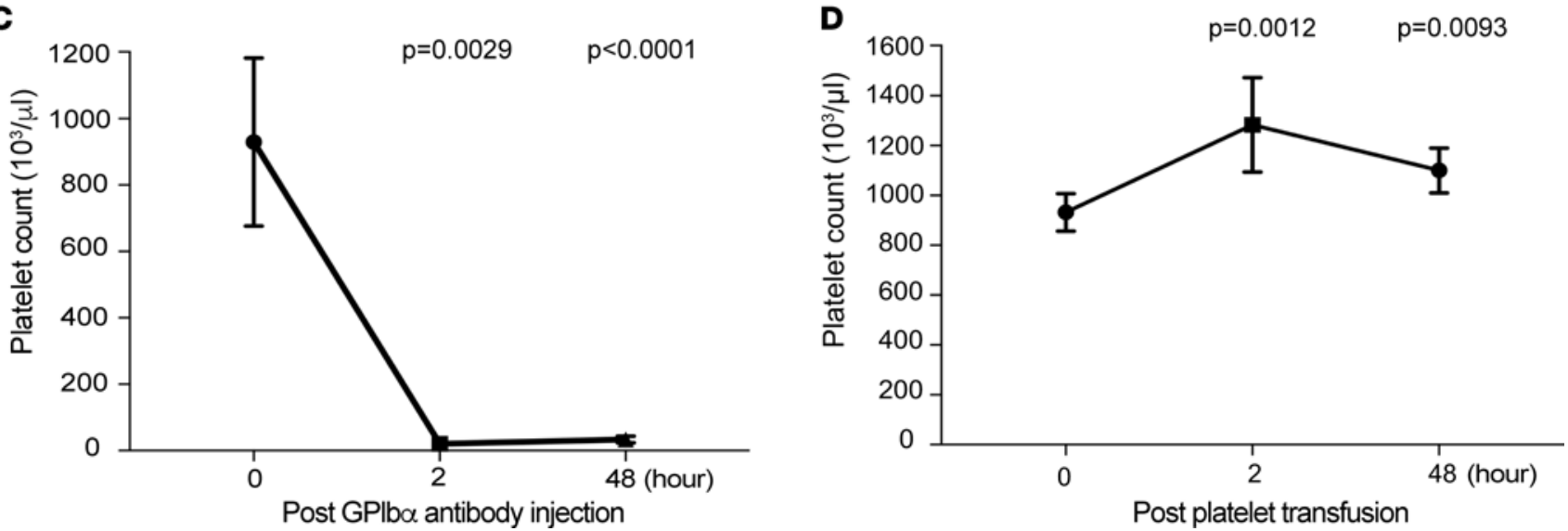

Figure 2. Platelet count in OIR and proof of concept. (A) Scheme of oxygen-induced retinopathy (OIR): C57BL/6 mouse pups are exposed to $75 \%$ oxygen from P7-P12 to induce retinal vessel loss and are returned to room air at P12-P17. Below the scheme, 2 representative retinal whole-mount images, including magnification area, from normoxia and OIR P17 pups stained with lectin (see magnification below). P17 OIR pups have pathologic neovascularization compared with normoxia pups. (B) Platelet counts in mouse pups at P7, P12, and P17 with (red) $(n=10)$ or without (blue) ( $n=6)$ OIR. Platelet count was reduced at P17 OIR $(P=0.0084)$. (C) Platelet counts were measured in whole blood after 0,2 , and 48 hours after anti-CPIb $\alpha$ antibody injection. A significant decrease in platelet count was seen at 2 and 48 hours after platelet depletion $(P=0.0029$ and $P<0.0001)$. (D) Platelet transfusion proof of concept. WT neonatal mice were retro-orbitally transfused with $10 \mu \mathrm{l}$ per gram of mouse of $5.0 \times 10^{6}$ platelets $/ \mu \mathrm{l}$. Total platelet counts were measured before transfusion (time 0 ), as well as 2 and 48 hours after transfusion. The platelet count increased by $42.3 \%$ after 2 hours $(P=0.0012)$. The initial increase was cleared by $58.6 \%$ after 48 hours but was still significantly increased compared with time $0(P=0.0093)$. Data represent mean \pm SD. Two-tailed unpaired $t$ test.

Platelet count in OIR model showed that platelet depletion with GPlba antibody decreased and platelet transfusion increased platelet counts in mice. In mice, platelet counts reached near-adult levels at P12 (mean: 1,266 $\times 10^{3} / \mu 1$; range: $800-1,500 \times 10^{3} / \mu 1$ ). In OIR, platelet counts were significantly lower compared with the normoxia control group at P17, the time of maximal NV $(P=0.0084$; Figure $2 \mathrm{~B})$. A platelet-depletion proof-of-concept study showed a $97.8 \%$ decrease in platelet count $(P=0.0029) 2$ hours after GPlb $\alpha$ antibody injection, which persisted for 48 hours (Figure 2C). Retro-orbital transfusion with $10 \mu 1$ per gram body weight of a platelet suspension containing $5.0 \times 10^{6}$ platelets $/ \mu 1$ led to a $42.3 \%$ increase in platelet concentration at 2 hours $(P=0.0012)$. The initial increase was cleared by $58.6 \%$ after 48 hours, but the platelet count was still significantly higher compared with time $0(P=0.0093$; Figure $2 \mathrm{D})$. 
A
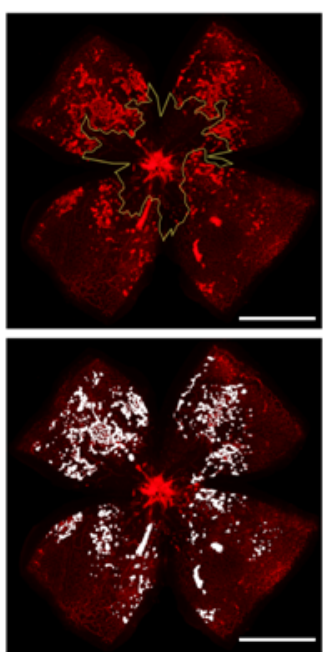

GPIb $\alpha$

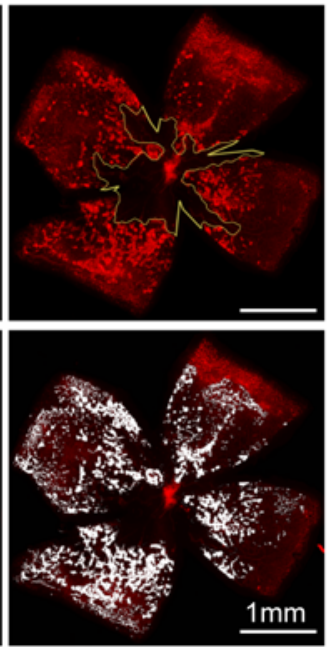

B

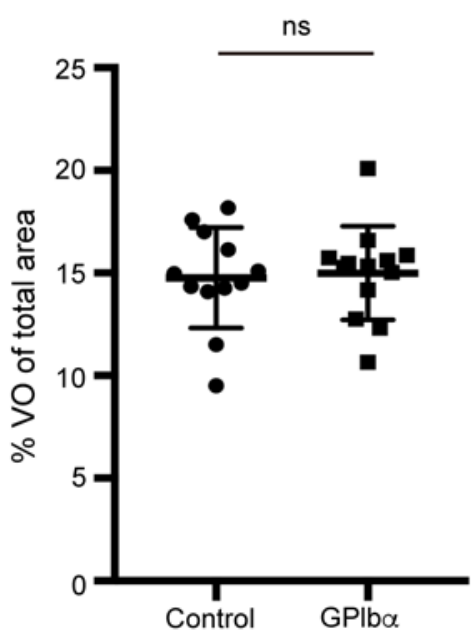

Neovascularization

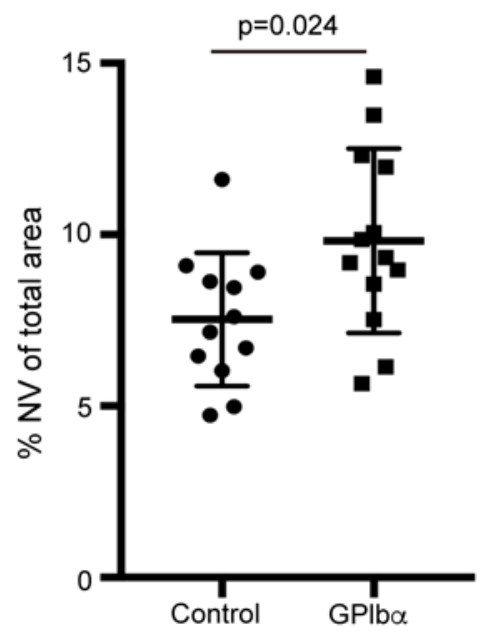

C

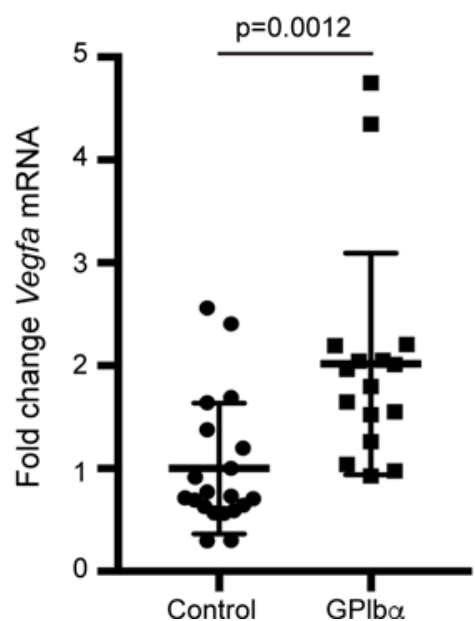

D

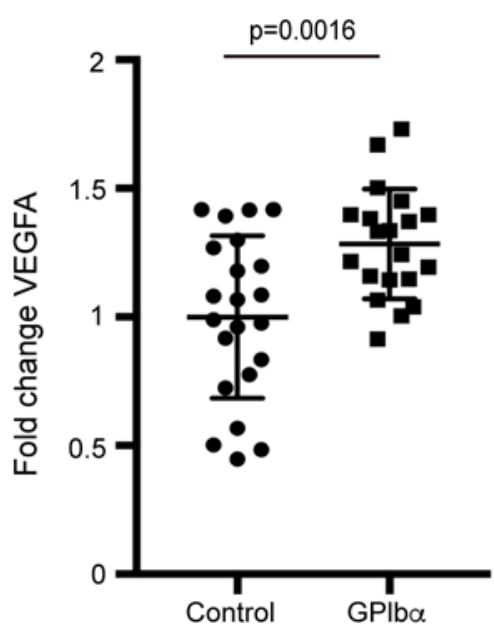

E

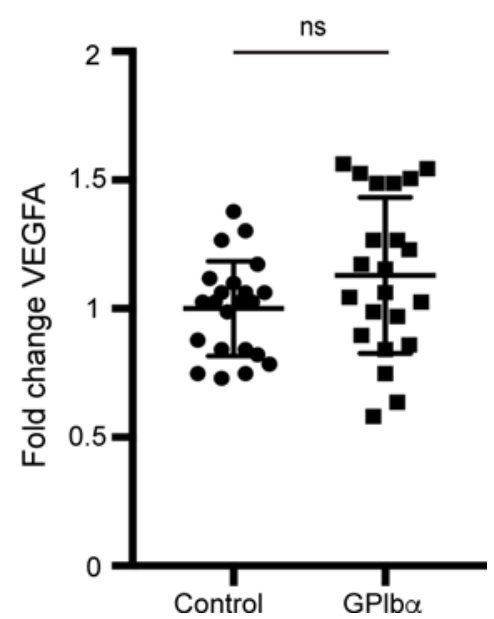

Figure 3. Platelet depletion in the OIR mouse model. Pups with OIR were platelet depleted with anti-GPIb $\alpha$ antibody $(n=13$ eyes) or PBS control on P15 ( $n=12$ eyes). (A) Representative images show the area of avascular retina or vaso-obliteration (VO) (outlined in yellow lines) in whole-mounted retina of treated (upper right panel) and control (upper left panel) pups. White areas illustrate neovascularization (NV) in treated (lower right) versus control (lower left) pups. (B) The VO area was unchanged in control pups vs. platelet-depleted pups $(P=0.81)$. The NV area was significantly higher in platelet-depleted pups vs. control $(P=0.024)$. (C) Measurement of Vegfa mRNA expression in the retina at P17. Vegfa mRNA expression was 2 -fold increased after platelet depletion ( $n=8$ eyes; 2 technical repeats) compared with the control group ( $n=10$ eyes; 2 technical repeats) $(P=0.0012)$. (D) ELISA VEGFA protein analysis of retina at P17. VEGFA protein expression was increased after platelet depletion ( $n=10$ eyes; 2 technical repeats) compared with the control group ( $n=11$ eyes; 2 technical repeats) $(P=0.0016)$. (E) ELISA measurements of plasma VEGFA protein at P17 revealed no significant difference after platelet depletion ( $n=11 ; 2$ technical repeats) compared with the control group ( $n=11 ; 2$ technical repeats) $(P=0.095)$. Unpaired $t$ test was used for statistical analysis. Data represent mean \pm SD.

At P15 in OIR (onset of neovascular tuft formation), platelet depletion increased and platelet transfusion decreased $N V$. In the OIR model, platelet depletion at P15 (the onset of neovascular tuft formation) increased proliferative retinopathy by $30.4 \%(P=0.024)$ (Figure $3, \mathrm{~A}$ and $\mathrm{B})$. Vaso-obliteration (VO) was not affected (Figure 3, A and B). The increased NV was associated with increased neural retina Vegfa mRNA and VEGFA protein expression $(P=0.0012$ and $P=0.0016)$, while VEGFA protein levels in the plasma were unchanged (Figure 3, C-E). In contrast to platelet depletion, platelet transfusions given at the same time point $(\mathrm{P} 15+\mathrm{P} 16)$ reduced $\mathrm{NV}$ by $19.3 \%(P=0.008)$ (Figure $4, \mathrm{~A}$ and $\mathrm{B})$. This suppression of NV was associated with decreased neural retina Vegfa mRNA and VEGFA protein expression $(P<0.0001$ and $P=0.011$ ) (Figure $4, C$ and D). Platelet depletion and platelet transfusion had no significant impact on VEGFA protein plasma levels (Figure 4E). 
A
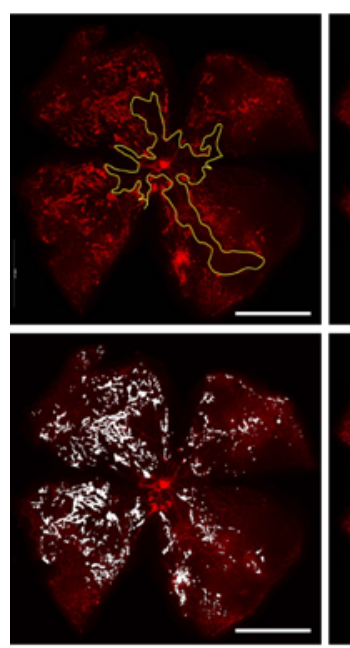

B
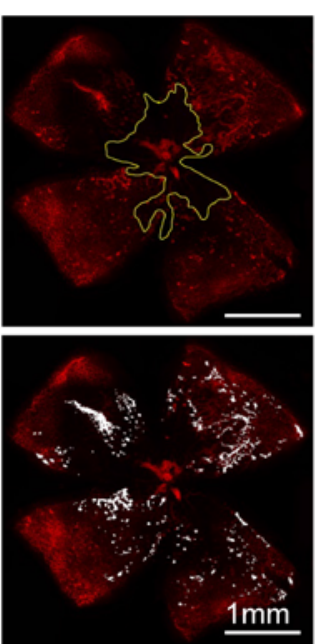

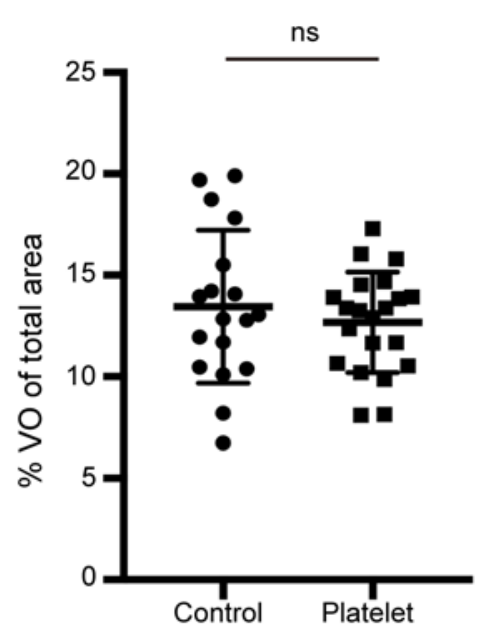

Neovascularization

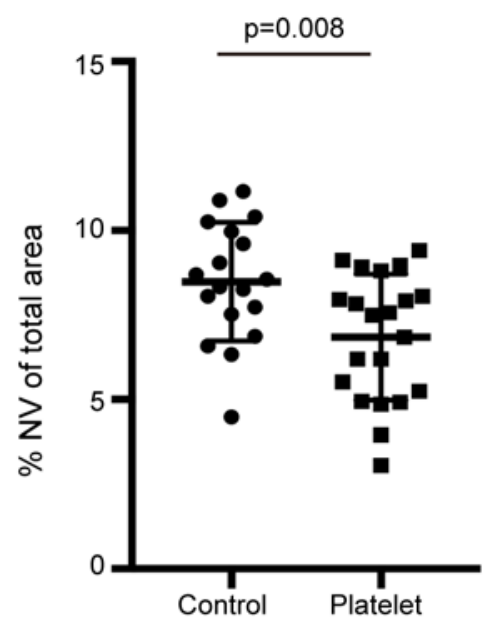

C Retina

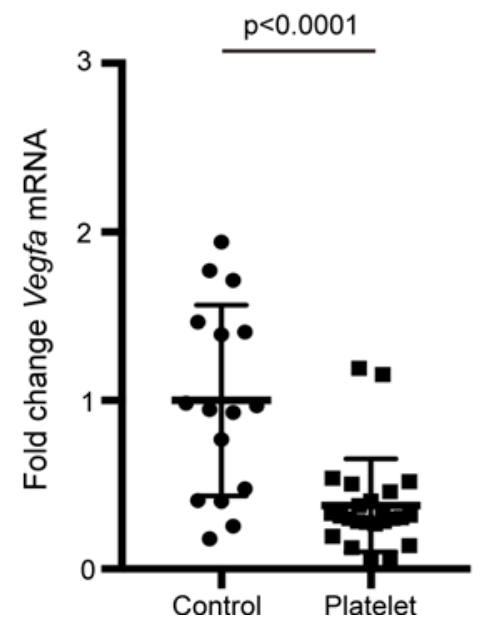

D

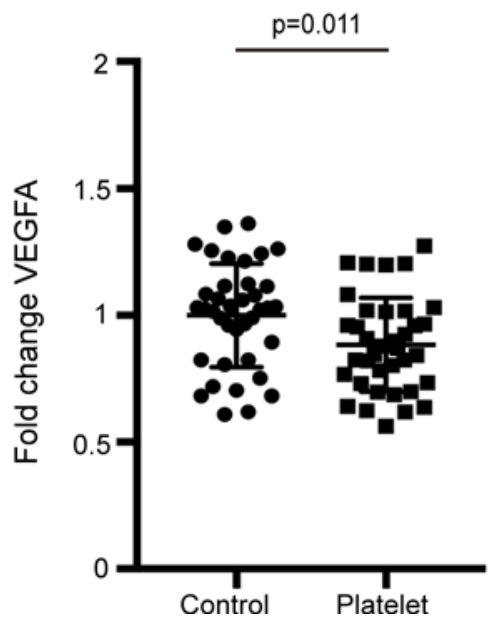

E

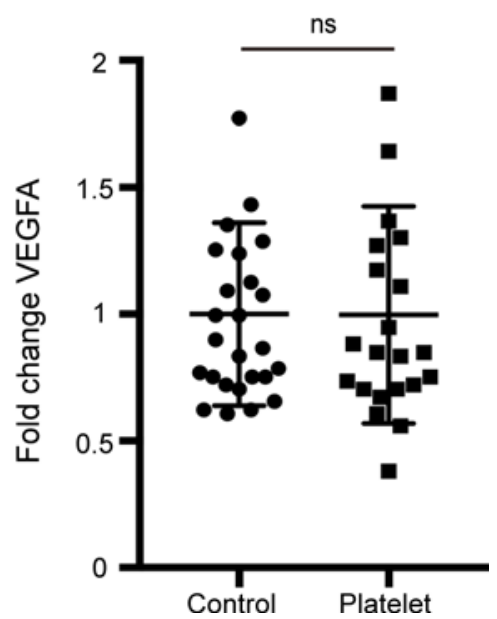

Figure 4. Platelet transfusion in the OIR mouse model. On P15 and P16, mouse pups with OIR were injected retro-orbitally with isolated concentrated washed platelets $\left(2.6 \times 10^{6}\right.$ to $3.3 \times 10^{6}$ platelets/ $\mu$ l) from adult C57/BI6 mice ( $n=21$ eyes) or PBS ( $n=18$ eyes). (A) Representative images show the area of avascular retina or vaso-obliteration (VO) (outlined in yellow lines) in whole-mounted retina of treated (upper right panel) and control (upper left panel) pups. White areas illustrate neovascularization (NV) in treated (lower right) vs. control (lower left) pups. (B) The VO area was unchanged in control pups vs. platelet transfused pups $(P=0.446)$. The NV area was significantly decreased in platelet transfused pups vs. control $(P=0.008)$. (C) Measurement of Vegfa mRNA expression in the retina at P17. Vegfa mRNA expression was decreased after platelet transfusion ( $n=12$ eyes; 2 technical repeats) compared with the control group ( $n=8$ eyes; 2 technical repeats) $(P<0.0001)$. (D) ELISA VEGFA protein analysis of retina at P17. VEGFA protein expression was decreased after platelet transfusion ( $n=19$ eyes; 2 technical repeats) compared with the control group ( $n=19$ eyes; 2 technical repeats) $(P=0.011)$. (E) ELISA measurements of plasma VEGFA protein at P17 revealed no significant difference after platelet depletion ( $n=11 ; 2$ technical repeats) compared with the control group ( $n=12 ; 2$ technical repeats) $(P=0.84)$. Unpaired $t$ test was used for statistical analysis. Data represent mean \pm SD.

Resting platelet, but not degranulated platelet transfusions, suppressed $N V$. To determine whether the observed effects of platelets were dependent upon the release of their granule content, we generated degranulated platelets with no $\alpha$-granules by exposing washed platelets to thrombin (15-17). P-selectin is stored in secretory granules of platelets and is rapidly expressed on the surface upon activation. The expression of P-selectin, therefore, reflects the degranulation of platelets (15). Activation with $1 \mathrm{U} / \mathrm{ml}$ thrombin led to P-selectin expression in $93 \%$ of platelets (versus $0.7 \%$ of unstimulated resting platelets), indicating degranulation of most platelets (Figure 5 , $\mathrm{A}$ and $\mathrm{B})$. To confirm comparable clearance rates, degranulated and resting $\mathrm{GFP}^{+}$platelets were followed over time after transfusion in neonatal P17 pups. No significant difference in clearance was seen between groups (Figure 5C). In the OIR model, NV was significantly reduced in the resting platelet transfusion group compared with the degranulated platelet transfusion group $(P=0.039)$. VO was unchanged (Figure 5D). 
A

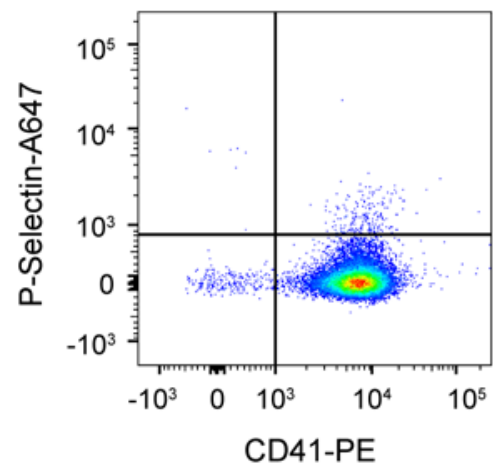

C

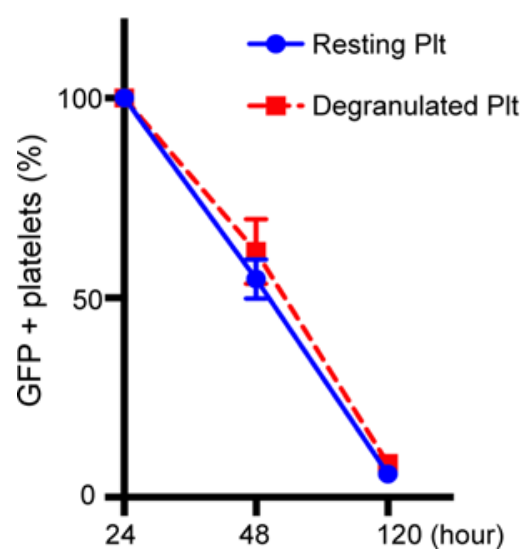

Degranulated platelets

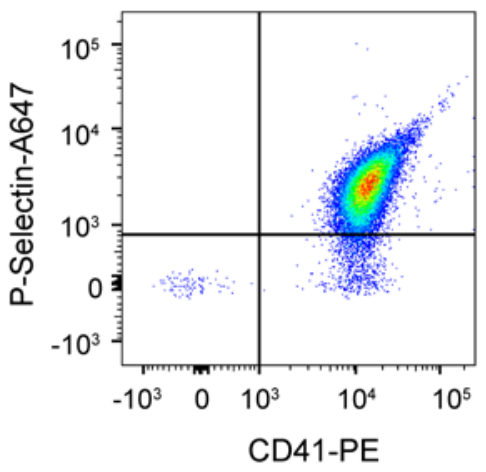

D

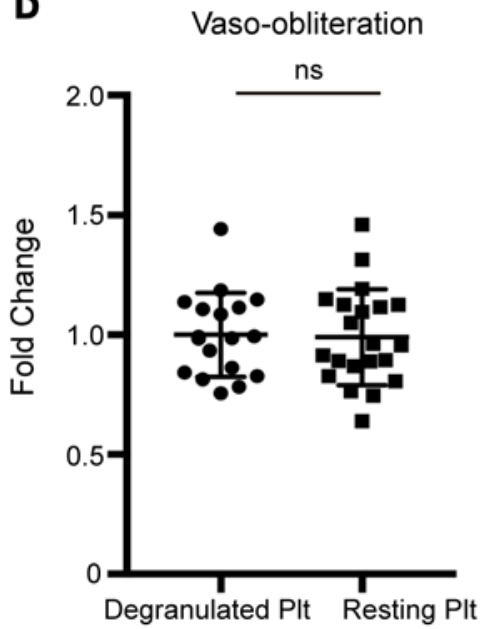

B P-selectin expression

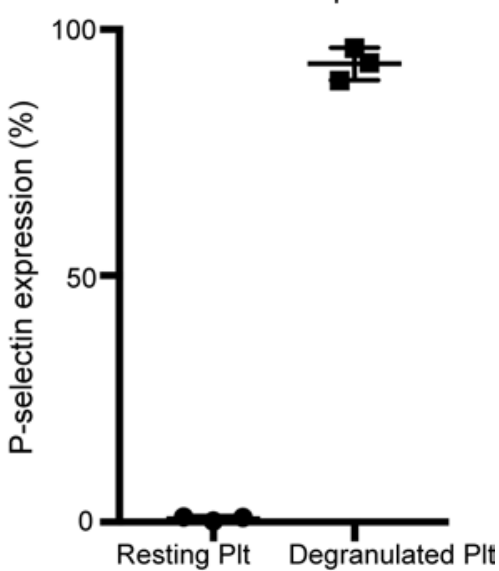

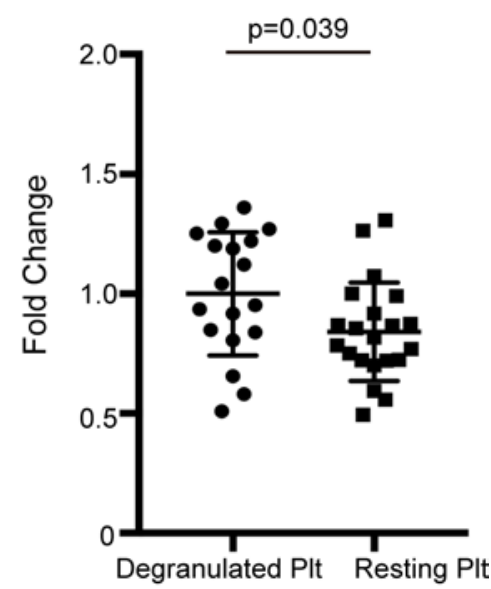

Figure 5. Comparison of neovascularization and vaso-obliteration in OIR using degranulated versus resting platelets. Platelet degranulation was achieved by stimulating platelet suspensions for 10 minutes with $1 \mathrm{U} / \mathrm{ml}$ thrombin. Control resting platelets were treated exactly the same except thrombin activation. (A) Representative FACS dot plot images of P-selectin and CD41 expression on degranulated (right) and resting platelets (left). (B) P-selectin expression on degranulated platelets was significantly increased compared with resting platelets, demonstrating that platelets were effectively degranulated through thrombin stimulation ( $93 \%$ vs. $0.737 \% ; P<0.0001$ ). (C) Comparison of degranulated and resting platelet clearance over time was determined by measuring GFP ${ }^{+}$fluorescence in platelets from GFP' donor mice through FACS analysis. Time 0 was defined as 24 hours after platelet transfusion. In vivo clearance of GFP' degranulated and resting platelets was unchanged. (D) Neovascularization was significantly decreased in the resting platelet transfusion group ( $n=21$ eyes) compared with the degranulated platelet transfusion group ( $n=18$ eyes; $P=0.039$ ). Vaso-obliteration was unchanged $(P=0.87)$. Unpaired $t$ test was used for statistical analysis. Data represent mean $\pm \mathrm{SD}$.

\section{Discussion}

We investigated longitudinal platelet counts in relation to the development of severe ROP clinically and experimentally (in a mouse model of ROP). We then evaluated the effects of platelet transfusions and depletion on proliferative ROP in OIR in mice. The most prominent factor associated with severe ROP in our retrospective analysis was any episode of thrombocytopenia at $\geq 30$ weeks PMA, even after adjusting for well-known confounding factors such as GA, NEC, and sepsis. This is in agreement with 2 case-control studies and a longitudinal study reporting that thrombocytopenia was independently associated with severe ROP requiring treatment $(11,12,14)$. In our study, all infants with severe ROP requiring laser treatment with any thrombocytopenia at $\geq 30$ weeks PMA also had some thrombocytopenia in the initial vessel loss phase of ROP ( $<30$ weeks PMA), indicating a possible multi-hit pattern (13).

In the oxygen-induced retinopathy mouse model, the reduction of vasoproliferation at the beginning of neovascular tuft formation (P15-P16) after platelet transfusions was in agreement with a report of regression of severe ROP in human preterm neonates after platelet transfusions during proliferative retinopathy (10). The increased NV after platelet depletion supports the hypothesis and is in line with the clinical correlation seen in our retrospective analysis. 
The effect of VEGFA in the oxygen induced retinopathy model (OIR) has been intensively investigated and was identified as the most important mediator of pathological NV in the disease. In phase I of the ROP model of OIR, high oxygen levels suppress retinal VEGFA expression, which leads to central VO. Upon return to room air at P12, hypoxia in the central avascular area leads to upregulation of VEGFA, primarily by Müller cells and astrocytes (18). The rapid increase in retinal VEGFA leads to NV into the vitreous starting at P14-P15. In our murine study, platelet depletion led to an increase, and platelet transfusion to a decrease, in retinal Vegfa mRNA and VEGFA protein expression. The exact mechanism of how platelets in the bloodstream influence retinal VEGFA expression is unclear. However, Müller glia cells (where most hypoxia-induced VEGFA is expressed in OIR) have close interactions with both vessels and neurons, and they play an important role in the homeostasis of the retina (19). It is therefore possible that an antiangiogenic stimulus released from platelets is communicated through endothelial cells to Müller cells, resulting in downregulation of Vegfa mRNA and VEGFA protein expression.

Platelets have numerous bioactive molecules stored in 3 types of secretory granules including $\alpha$-granules, dense granules, and lysosomes, enabling them to exert a wide range of effects on the surrounding microenvironment (20). $\alpha$-Granules are the most abundant and contain over 100 bioactive proteins including various pro- and antiangiogenic factors such as angiostatin, endostatin, platelet factor- 4 , thrombospondin- 1 , $\alpha_{2}$-macroglobulin, and plasminogen activator inhibitor $1(21,22)$. Even though platelets contain pro- and antiangiogenic factors, the reported effect of platelets on endothelial cells has mainly been proangiogenic or proproliferative (23-27). Pipili-Synetos et al. (23) showed a proliferative effect of platelets on HUVEC in vitro for the first time in 1998. Enhanced endothelial cell tube formation was seen in a dose-dependent manner with stimulation with resting platelets but, interestingly, not with $\alpha$-thrombin-stimulated platelet supernatant. Pipili-Synetos and colleagues (23) concluded that a direct interaction between endothelium and platelets is necessary to promote the proproliferative effect. Further, data from clinical and experimental cancer research also supports a stimulatory effect of platelet on tumor angiogenesis (28). Nevertheless, the observation by Italiano et al. that pro- and antiangiogenic proteins are separated in specific subpopulations of $\alpha$-granules inspired the hypothesis that platelets can locally promote or inhibit angiogenesis through controlled granule release (5). A follow-up investigation by the same group showed that angiogenic proteins can be differentially secreted in different in vitro conditions. Stimulation of washed platelets with the platelet activators ADP and Thromboxane $\mathrm{A}_{2}\left(\mathrm{TXA}_{2}\right)$ led to a differential release of VEGF (stimulant of vessel growth) or endostatin (inhibitor of vessel growth), respectively. In accord, a divergent angiogenic effect was seen in a tube formation assay after stimulation with platelet releasate generated by ADP and $\mathrm{TXA}_{2}$, demonstrating that platelets can stimulate or suppress angiogenesis in vitro, depending on conditions (29). The in vitro data is further supported by Ma et al. studies investigating the effect of platelets on gastric ulcer healing in rats in vivo (27). Treatment with the ADP antagonist Ticlopidine increased serum endostatin and decreased VEGF levels, which resulted in decreased gastric ulcer healing. In the same study, serum from Ticlopidine-treated rats reduced angiogenesis in a sprouting assay in vitro (27).

Our murine study is, to our knowledge, the first study showing a reduction of NV in vivo by platelets. We hypothesize that the unique microenvironment in the pathological neovascular tuft area at P15-P17 leads to a controlled release of antiangiogenic factors, resulting in the suppression of neovascular tuft formation and downregulation of retinal VEGFA expression through neurovascular crosstalk. Platelet transfusion or platelet depletion did not affect the area of VO in the retina in the OIR model.

In order to distinguish whether platelet granule release or the platelet interaction with endothelium by itself causes the suppression of $\mathrm{NV}$, we generated degranulated platelets by activating washed platelets in vitro with thrombin. We compared the effect of degranulated versus resting platelets with intact granules and found that degranulated platelets, unlike resting platelets, did not affect NV. This supports the hypothesis that factors within the granules are important to suppress NV.

In order to more fully address the mechanism with respect to which platelet factors mediate the protective effect on ROP, we need mice with single or possibly multiple genetic depletions of factors specifically in platelets. These mice need to be generated, which will take considerable time and will be part of future investigations in our laboratories.

Overall, our findings suggest a local interactive effect of platelets specifically on endothelial cells in neovascular tufts that leads to direct inhibition of $\mathrm{NV}$, as well as a secondary regulation of VEGFA expression in the neural retina, ultimately controlling NV in ROP. Our data suggest that platelets are important for normal fetal/neonatal vascular development and that, during the proliferative phase of ROP, low platelet 
counts are detrimental, likely due to a lack of platelet factors as yet to be determined. The risks and benefits of platelet transfusions in preterm infants, a population with unique physiology and pathological processes, are in general poorly understood $(6,30)$, and transfusions should be carefully considered.

In summary, clinical thrombocytopenia at PMA $>30$ weeks (at the onset of proliferative retinopathy) is a prominent factor associated with severe ROP requiring treatment. In addition, in the mouse model of ROP at the onset of neovascular tuft formation, platelet depletion increases retinopathy while platelet transfusion decreases retinopathy.

\section{Methods}

Patients. We retrospectively reviewed 237 infants born at GA $<27$ weeks and screened for ROP in Stockholm from 2008-2011 $(n=176)$ and Gothenburg from 2013-2015 $(n=61)$. International guidelines for ROP classification and ROP treatment were followed $(31,32)$. We retrieved ROP data from the Swedish national register for ROP (SWEDROP) (33). We defined the initial phase of ROP, with suppressed vascularization, as $<30$ weeks PMA and the later proliferative phase as 30-36 weeks PMA. Sepsis was defined as a positive blood culture and/or clinical signs and C-reactive protein $>20 \mathrm{mg} / 1$. Platelet counts and platelet transfusions from birth until 36 weeks PMA were recorded; 35 infants were excluded for lack of platelet counts until at least 30 weeks PMA. Thrombocytopenia was defined as any platelet count $<100 \times 10^{9} / 1$. Mean platelet count per day was assessed for each infant based on these data, and a mean weekly platelet count was calculated for each infant.

OIR. The mouse OIR model was used as described (34-36). Briefly, C57BL/6 mouse pups (with nursing dams) (The Jackson Laboratory) are exposed to 75\% oxygen from P7-P12 to induce retinal vessel loss and returned to room air at P12-P17, when hypoxic retina stimulates NV. Mice with body weight under $5.5 \mathrm{~g}$ or over $7.8 \mathrm{~g}$ were excluded in this study. Maximum retinal NV and remaining VO at P17 was quantified (Figure 2A).

Mouse platelet transfusions. Blood was collected from adult C57BL/6 mice through retro-orbital bleeds in enoxaparin $(0.2 \mu \mathrm{g} / \mathrm{ml})$ under isoflurane anesthesia. Platelet-rich plasma (PRP) was collected after 2 centrifugation cycles of $300 \mathrm{~g}$ for 7 minutes at room temperature (RT). PRP was pelleted at $700 \mathrm{~g}$ for 5 minutes in the presence of prostacyclin ( $\mathrm{PGI}_{2}$; MilliporeSigma) $(0.1 \mu \mathrm{g} / \mathrm{ml})$ and apyrase $(0.02 \mathrm{U} /$ $\mathrm{ml}$; MilliporeSigma). The platelet pellet was resuspended in modified Tyrode-HEPES buffer (134 mM $\mathrm{NaCl}$ [Thermo Fisher Scientific], $0.34 \mathrm{mM} \mathrm{Na}_{2} \mathrm{HPO}_{4}$ [Thermo Fisher Scientific], $2.9 \mathrm{mM} \mathrm{KCl}$ [Thermo Fisher Scientific], $12 \mathrm{mM} \mathrm{NaHCO}_{3}$ [MP Biomedicals], $5 \mathrm{mM}$ HEPES [Thermo Fisher Scientific], $1 \mathrm{mM}$ $\mathrm{MgCl}_{2}$ [Thermo Fisher Scientific], $5 \mathrm{mM}$ glucose [MilliporeSigma], 0.35\% BSA [MilliporeSigma], $\mathrm{pH}$ 7.4) containing apyrase and $\mathrm{PGI}_{2}$ and left for 10 minutes at $37^{\circ} \mathrm{C}$. Platelets were pelleted at $700 \mathrm{~g}$ for 5 minutes, resuspended in Tyrode's buffer containing apyrase to a concentration of $2.6 \times 10^{6}$ to $3.3 \times 10^{6}$ platelets $/ \mu 1$, and left for 30 minutes at $37^{\circ} \mathrm{C}$ before use. Resting state of platelets was confirmed by flow cytometry (Supplemental Figure 1; supplemental material available online with this article; https://doi. org/10.1172/jci.insight.99448DS1). Recipient mice were transfused with $10 \mu 1$ per gram body weight of this washed platelet suspension or vehicle through retro-orbital injection on days P15 and P16.

Platelet degranulation. For degranulation, platelet suspensions were stimulated for 10 minutes with $1 \mathrm{U} / \mathrm{ml}$ thrombin (Chrono-log) under stirring conditions at $37^{\circ} \mathrm{C}$ in a Chrono-log platelet aggregometer in the presence of $2 \mathrm{mM}$ EDTA (IBI Scientific) to avoid aggregation. The reaction was stopped by the addition of hirudin (Pentapharm), as previously described (15). Resting platelet suspensions were treated in the same way, with the exception that an equal volume of Tyrode's buffer was added instead of thrombin. Platelet suspensions were spun down at $700 \mathrm{~g}$, the supernatant was removed, and platelet pellets were resuspended in sterile saline to a concentration of $2.6 \times 10^{6}$ to $3.3 \times 10^{6}$ platelets $/ \mu 1$ and left for 30 minutes at $37^{\circ} \mathrm{C}$ before use. Platelet degranulation was confirmed by flow cytometry analysis of P-selectin expression on the platelet surface. Briefly, 20,000 platelet/ $\mu 1$ in a final volume of $40 \mu 1$ were stained for 15 minutes at RT with an AF647-coupled anti-P-selectin antibody (BD Pharmingen, catalog 563674) and a PE-coupled anti-CD41 antibody (BD Pharmingen, catalog 558040). The reaction was stopped by the addition of $300 \mu \mathrm{PBS}$ and data obtained on a FACS Canto (BD Biosciences). Comparable platelet lifespan rates in circulation between platelet preparations were ensured by measuring the percentage of $\mathrm{GFP}^{+}$degranulated and resting platelets in whole blood after transfusion in WT mice through FACS analysis. Recipient mice were transfused with $10 \mu 1$ per gram body weight of degranulated or resting platelets through retro-orbital injection on days P15 and P16. 
Platelet depletion in mouse pups. To deplete platelets in mice with OIR, $2 \mu \mathrm{g}$ per gram body weight antiGPIb $\alpha$ (CD42b) antibody (R300, Emfret) in sterile PBS was injected i.p. in P15 mice. Littermates were injected with PBS as controls. The phenotype experiment was also repeated with IgG as control. A comparable effect was seen with IgG or PBS as control (Supplemental Figure 2.)

Complete cell counts. For complete cell counts, blood samples were diluted 1:20 with Cellpack solution supplemented with EDTA and PGE $_{1}$ (MilliporeSigma) and counted using a Sysmex XT-2000i automatic hematology analyzer (Sysmex).

Protein and RNA analyses. Following enucleation, retinas were immediately dissected, washed in PBS, and cleaned from the remaining vitreous. The retina was cut in half for RNA and protein analyses. Total RNA was extracted from mouse retina using RNeasy kit (Qiagen) and reverse-transcribed with SuperScript III Reverse Transcriptase (Thermo Fisher Scientific). A SYBR-green based real-time PCR was used for quantification. VEGFA plasma and retina protein levels were quantified using standard mouse VEGF ELISA Kit (Quantikine ELISA, R\&D Systems). Briefly, retinal homogenized in PBS by sonication, centrifuged for 5 minutes at 5,000 $\mathrm{g}$ and assayed according to standard protocol. Blood was collected after enucleation, transferred to an EDTA-coated tube, and centrifuged for 20 minutes at 2,000 g. Plasma supernatant was diluted 1:5 and assayed according to standard protocol. A microplate reader set at $450 \mathrm{~nm}$ with wavelength correction for $540 \mathrm{~nm}$ was used for optical density measurement.

Animals. WT C57BL/6 mice from the Jackson Laboratory were used for all platelet transfusion and depletion experiments. For proof-of-concept experiments, $129 \mathrm{~S} 6$ mice from Taconic were included in the analysis. $\mathrm{GFP}^{+}$platelets were isolated from adult C57BL $/ 6^{\mathrm{Tg}(\mathrm{UBC}-\mathrm{GFP}) 30 \mathrm{Scha} / \mathrm{J}}$ mice (strain no. 004353; the Jackson Laboratory). Both sexes were included in this study.

Statistics. Statistical analysis was performed using GraphPad Prism 6 and IBM SPSS Statistics 24 for Microsoft Windows (IBM). For the preclinical study, a 2-tailed unpaired $t$ test was used for comparison between 2 groups. In the clinical data analysis $\chi^{2}$ test and Mann-Whitney $U$ test was used for comparison between 2 groups. OR and adjusted OR were calculated using logistic regression analysis. A $P$ value below or equal 0.05 was considered as significant.

Study approval. The clinical study was approved by the hospital review boards and carried out in accordance with the 1964 Helsinki declaration and its later amendments or comparable ethical standards. All animal studies adhered to the Association for Research in Vision and Ophthalmology Statement for the Use of Animals in Ophthalmic and Vision Research and were approved by the Boston Children's Hospital IACUC. International guidelines for ROP classification and ROP treatment were followed $(31,32)$.

\section{Author contributions}

$\mathrm{PL}, \mathrm{RL}, \mathrm{BC}$, and GH contributed equally to this work. PL, GH, and AH designed and performed the clinical research, collected and analyzed data, and wrote the manuscript; BC, RL, YS, ZJL, MCSV, and LEHS designed and performed the preclinical research, collected and analyzed data, and wrote the manuscript. SC, AP, RD, VL, and JC collected and analyzed data. SK, GH, CM, CL, and BH provided advice, assisted with data collection and statistical analyzes, and approved the manuscript.

\section{Acknowledgments}

We thank the board of SWEDROP and all the physicians that performed ROP screening in Sweden. This study was supported by the Herman Svensson Foundation, the Cronqvists Foundation, Linnéa and Josef Carlssons Foundation, HKH Kronprinsessan Lovisas förening för barnasjukvård/Stiftelsen Axel Tielmans Minnesfond, Stiftelsen Kronprinsessan Margaretas Arbetsnämnd för synskadade, De blindas vänner NIH (EY024864, EY017017, P01 HD18655, P01 HL046925, and HL69990), European Commission FP7 project 305485 PREVENT-ROP, the Lowy Medical Research Institute, and Deutsche Forschungsgemeinschaft (DFG; to BC [CA 1940/1-1] and RL). The Sysmex XT-2000i Automatic Veterinary Analyzer used to measure murine cell counts is a loan from Sysmex, Kobe, Japan, to MCSV's laboratory.

Address correspondence to: Ann Hellström, Institute of Neuroscience and Physiology, The Sahlgrenska Academy at University of Gothenburg, S-416 85 Gothenburg, Sweden. Phone: 46.768979196; Email: ann.hellstrom@medfak.gu.se. Or to: Lois E. H. Smith, Department of Ophthalmology, Boston Children's Hospital, Harvard Medical School, 300 Longwood Avenue, Boston Massachusetts 02115, USA. Phone: 1.617.355.6401; Email: 1ois.smith@childrens.harvard.edu. 
1. Gilbert C, et al. Characteristics of infants with severe retinopathy of prematurity in countries with low, moderate, and high levels of development: implications for screening programs. Pediatrics. 2005;115(5):e518-e525.

2. Hellstrom A, et al. Low IGF-I suppresses VEGF-survival signaling in retinal endothelial cells: direct correlation with clinical retinopathy of prematurity. Proc Natl Acad Sci USA. 2001;98(10):5804-5808.

3. Stone J, et al. Development of retinal vasculature is mediated by hypoxia-induced vascular endothelial growth factor (VEGF) expression by neuroglia. J Neurosci. 1995;15(7 Pt 1):4738-4747.

4. Folkman J. Angiogenesis: an organizing principle for drug discovery? Nat Rev Drug Discov. 2007;6(4):273-286.

5. Italiano JE, et al. Angiogenesis is regulated by a novel mechanism: pro- and antiangiogenic proteins are organized into separate platelet alpha granules and differentially released. Blood. 2008;111(3):1227-1233.

6. Sola-Visner M, Bercovitz RS. Neonatal Platelet Transfusions and Future Areas of Research. Transfus Med Rev. 2016;30(4):183-188.

7. Christensen RD, Yoder BA, Baer VL, Snow GL, Butler A. Early-Onset Neutropenia in Small-for-Gestational-Age Infants. Pediatrics. 2015;136(5):e1259-e1267.

8. Guida JD, Kunig AM, Leef KH, McKenzie SE, Paul DA. Platelet count and sepsis in very low birth weight neonates: is there an organism-specific response? Pediatrics. 2003;111(6 Pt 1):1411-1415.

9. Bonifacio L, Petrova A, Nanjundaswamy S, Mehta R. Thrombocytopenia related neonatal outcome in preterms. Indian J Pediatr. 2007;74(3):269-274.

10. Vinekar A, et al. Do platelets have a role in the pathogenesis of aggressive posterior retinopathy of prematurity? Retina (Philadelphia, Pa). 2010;30(4 Supp1):S20-S23.

11. Jensen AK, Ying GS, Huang J, Karp K, Quinn GE, Binenbaum G. Thrombocytopenia and retinopathy of prematurity. $J A A P O S$. 2011;15(1):e3-e4.

12. Jensen AK, Ying GS, Huang J, Quinn GE, Binenbaum G. Longitudinal study of the association between thrombocytopenia and retinopathy of prematurity. J AAPOS. 2018;22(2):119-123.

13. Lundgren $P$, et al. Aggressive Posterior Retinopathy of Prematurity Is Associated with Multiple Infectious Episodes and Thrombocytopenia. Neonatology. 2017;111(1):79-85.

14. Sancak S, Toptan HH, Gokmen Yildirim T, Karatekin G, Ovali F. THROMBOCYTOPENIA AS A RISK FACTOR FOR RETINOPATHY OF PREMATURITY [published online ahead of print January 23, 2018]. Retina. https://doi.org/10.1097/ IAE.0000000000002028.

15. Ho-Tin-Noé B, Goerge T, Cifuni SM, Duerschmied D, Wagner DD. Platelet granule secretion continuously prevents intratumor hemorrhage. Cancer Res. 2008;68(16):6851-6858.

16. Berger G, Hartwell DW, Wagner DD. P-Selectin and platelet clearance. Blood. 1998;92(11):4446-4452.

17. Michelson $\mathrm{AD}$, et al. In vivo tracking of platelets: circulating degranulated platelets rapidly lose surface $\mathrm{P}$-selectin but continue to circulate and function. Proc Natl Acad Sci USA. 1996;93(21):11877-11882.

18. Pierce EA, Avery RL, Foley ED, Aiello LP, Smith LE. Vascular endothelial growth factor/vascular permeability factor expression in a mouse model of retinal neovascularization. Proc Natl Acad Sci USA. 1995;92(3):905-909.

19. Toft-Kehler AK, Skytt DM, Kolko M. A Perspective on the Müller Cell-Neuron Metabolic Partnership in the Inner Retina. Mol Neurobiol. 2018;55(6):5353-5361.

20. Rendu F, Brohard-Bohn B. The platelet release reaction: granules' constituents, secretion and functions. Platelets. 2001;12(5):261-273.

21. Maynard DM, Heijnen HF, Horne MK, White JG, Gahl WA. Proteomic analysis of platelet alpha-granules using mass spectrometry. J Thromb Haemost. 2007;5(9):1945-1955.

22. Maynard DM, Heijnen HF, Gahl WA, Gunay-Aygun M. The $\alpha$-granule proteome: novel proteins in normal and ghost granules in gray platelet syndrome. J Thromb Haemost. 2010;8(8):1786-1796.

23. Pipili-Synetos E, Papadimitriou E, Maragoudakis ME. Evidence that platelets promote tube formation by endothelial cells on matrigel. Br J Pharmacol. 1998;125(6):1252-1257.

24. Packham IM, Watson SP, Bicknell R, Egginton S. In vivo evidence for platelet-induced physiological angiogenesis by a COX driven mechanism. PLoS One. 2014;9(9):e107503.

25. Kisucka J, et al. Platelets and platelet adhesion support angiogenesis while preventing excessive hemorrhage. Proc Natl Acad Sci USA. 2006;103(4):855-860.

26. Ma D, et al. Expression of free fatty acid receptor GPR40 in the central nervous system of adult monkeys. Neurosci Res. 2007;58(4):394-401.

27. Ma L, Elliott SN, Cirino G, Buret A, Ignarro LJ, Wallace JL. Platelets modulate gastric ulcer healing: role of endostatin and vascular endothelial growth factor release. Proc Natl Acad Sci USA. 2001;98(11):6470-6475.

28. Wojtukiewicz MZ, Sierko E, Hempel D, Tucker SC, Honn KV. Platelets and cancer angiogenesis nexus. Cancer Metastasis Rev. 2017;36(2):249-262.

29. Battinelli EM, Markens BA, Italiano JE. Release of angiogenesis regulatory proteins from platelet alpha granules: modulation of physiologic and pathologic angiogenesis. Blood. 2011;118(5):1359-1369.

30. Lieberman L, Bercovitz RS, Sholapur NS, Heddle NM, Stanworth SJ, Arnold DM. Platelet transfusions for critically ill patients with thrombocytopenia. Blood. 2014;123(8):1146-1151.

31. International Committee for the Classification of Retinopathy of Prematurity. The International Classification of Retinopathy of Prematurity revisited. Arch Ophthalmol. 2005;123(7):991-999.

32. Early Treatment For Retinopathy Of Prematurity Cooperative Group. Revised indications for the treatment of retinopathy of prematurity: results of the early treatment for retinopathy of prematurity randomized trial. Arch Ophthalmol. 2003;121(12):1684-1694.

33. Holmström GE, Hellström A, Jakobsson PG, Lundgren P, Tornqvist K, Wallin A. Swedish national register for retinopathy of prematurity (SWEDROP) and the evaluation of screening in Sweden. Arch Ophthalmol. 2012;130(11):1418-1424. 
34. Smith LE, et al. Oxygen-induced retinopathy in the mouse. Invest Ophthalmol Vis Sci. 1994;35(1):101-111.

35. Connor KM, et al. Quantification of oxygen-induced retinopathy in the mouse: a model of vessel loss, vessel regrowth and pathological angiogenesis. Nat Protoc. 2009;4(11):1565-1573.

36. Stahl A, et al. Computer-aided quantification of retinal neovascularization. Angiogenesis. 2009;12(3):297-301. 